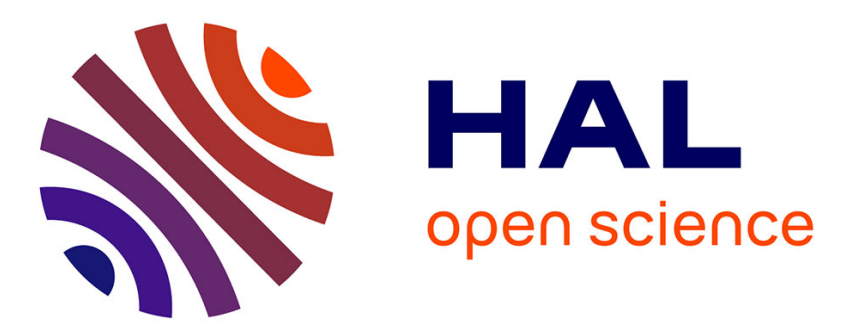

\title{
A nine point finite volume scheme for the simulation of diffusion in heterogeneous media
}

\author{
Léo Agélas, Robert Eymard, Raphaele Herbin
}

\section{To cite this version:}

Léo Agélas, Robert Eymard, Raphaele Herbin. A nine point finite volume scheme for the simulation of diffusion in heterogeneous media. Comptes rendus de l'Académie des sciences. Série I, Mathématique, 2009, 347 (11-12), pp.673-676. hal-00350139

\section{HAL Id: hal-00350139 \\ https://hal.science/hal-00350139}

Submitted on 6 Jan 2009

HAL is a multi-disciplinary open access archive for the deposit and dissemination of scientific research documents, whether they are published or not. The documents may come from teaching and research institutions in France or abroad, or from public or private research centers.
L'archive ouverte pluridisciplinaire HAL, est destinée au dépôt et à la diffusion de documents scientifiques de niveau recherche, publiés ou non, émanant des établissements d'enseignement et de recherche français ou étrangers, des laboratoires publics ou privés. 


\title{
A NINE-POINT FINITE VOLUME SCHEME FOR THE SIMULATION OF DIFFUSION IN HETEROGENEOUS MEDIA
}

\author{
LÉO AGELAS, ROBERT EYMARD, AND RAPHAÈLE HERBIN
}

\begin{abstract}
We propose a cell-centred symmetric scheme which combines the advantages of MPFA (multi point flux approximation) schemes such as the $\mathrm{L}$ or the $\mathrm{O}$ scheme and of hybrid schemes: it may be used on general non conforming meshes, it yields a 9-point stencil on two-dimensional quadrangular meshes, it takes into account the heterogeneous diffusion matrix, and it is coercive and convergent. The scheme relies on the use of special points, called harmonic averaging points, located at the interfaces of heterogeneity.
\end{abstract}

\section{INTRODUCTION}

The aim of this paper is to provide an approximation of the unique weak solution to the diffusion problem $-\operatorname{div}(\Lambda(\boldsymbol{x}) \nabla u)=f$ in $\Omega$ with boundary condition $u=0$ on $\partial \Omega$, where we denote by $\partial \Omega=\bar{\Omega} \backslash \Omega$ the boundary of the domain $\Omega$. Hence we wish to approximate

$$
u \in H_{0}^{1}(\Omega) \text { and } \forall v \in H_{0}^{1}(\Omega), \int_{\Omega} \Lambda(\boldsymbol{x}) \nabla u(\boldsymbol{x}) \cdot \nabla v(\boldsymbol{x}) \mathrm{d} \boldsymbol{x}=\int_{\Omega} f(\boldsymbol{x}) v(\boldsymbol{x}) \mathrm{d} \boldsymbol{x} .
$$

We assume that $\Omega$ is an open bounded connected polygonal subset of $\mathbb{R}^{2}$ (the adaptation of the present paper to the three-dimensional case will be the subject of future work), $\Lambda$ is a measurable function from $\Omega$ to $\mathcal{M}_{d}(\mathbb{R})$ with $d=2$, where we denote by $\mathcal{M}_{d}(\mathbb{R})$ the set of $d \times d$ matrices, such that for a.e. $\boldsymbol{x} \in \Omega, \Lambda(\boldsymbol{x})$ is symmetric, and such that the set of its eigenvalues is included in $[\underline{\lambda}, \bar{\lambda}]$, with $\underline{\lambda}$ and $\bar{\lambda} \in \mathbb{R}$ satisfying $0<\underline{\lambda} \leq \bar{\lambda}$, and $f \in L^{2}(\Omega)$. Our quest is motivated in particular by the numerical simulation of complex flows in porous media, which includes coupling with thermodynamics and/or chemistry; because of these complex couplings, the discretization method is often chosen to be cell-centred in industrial codes. Although several schemes were recently proposed see e.g. [7] and references therein, there is yet no "ultimate" scheme, i.e. a centred scheme with small stencil, which respects the physical bounds and yields good approximations even on nonconforming distorted meshes and with a sharp contrast in the permeabilities (or diffusion coefficients).

The new scheme which we introduce here is designed in the framework of this quest, and has the following characteristics:

(1) it may be used on any polygonal non-conforming mesh,

(2) it provides the exact solution if $\Lambda$ is piecewise constant in polygonal subdomains and $u$ is affine in each of these subdomains (this property is sought in the multipoint flux approximation schemes given for instance in [1]),

(3) it leads to a nine-point scheme in the case of quadrilateral meshes which are not too distorted (in a sense involving the diffusion matrix $\Lambda$ ),

This work was supported by Groupement de Recherche MOMAS, PACEN/CNRS. 


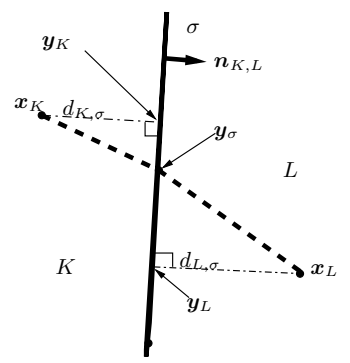

(a) The harmonic averaging point

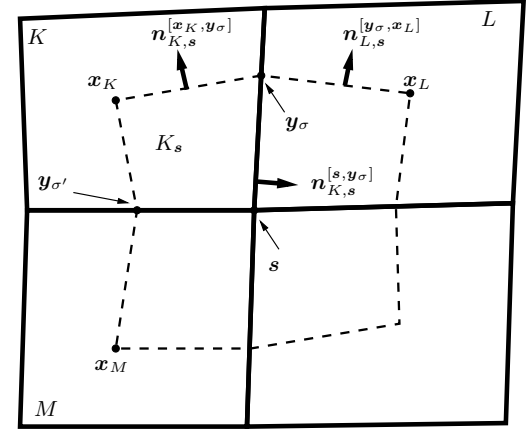

(b) Description of the mesh

\section{FiguRE 1}

(4) it is symmetric and coercive with respect to an adequate discrete norm, and therefore a convergence proof holds.

\section{HARMONiC AVERAGing POINTS}

Consider two domains $K$ and $L$ of $\mathbb{R}^{2}$ with different diffusion matrices (or permeabilities) $\Lambda_{K}$ and $\Lambda_{L}$, separated by a planar interface $\sigma$, and let $\boldsymbol{x}_{K} \in K$ and $\boldsymbol{x}_{L} \in L$. In order to obtain a scheme with smallest possible stencil, we seek some point in $\sigma$ where the value of any piecewise affine solution $u$ to (1) can be expressed as a linear combination of $u\left(\boldsymbol{x}_{K}\right)$ and $u\left(\boldsymbol{x}_{L}\right)$ only. We show in the next lemma that such a point always exists in the hyperplane containing $\sigma$.

Lemma 2.1. Let $\sigma$ be a hyperplane of $\mathbb{R}^{d}$, with $d \in \mathbb{N}^{\star}$ and let $K, L$ be the two open half-spaces with the common boundary $\sigma$. Let $\Lambda_{K} \in \mathcal{M}_{d}(\mathbb{R})$ and $\Lambda_{L} \in \mathcal{M}_{d}(\mathbb{R})$ be two given symmetric definite positive matrices, let $\boldsymbol{n}_{K L}$ be the unit vector normal to $\sigma$ oriented from $K$ to $L, \boldsymbol{x}_{K} \in K$ and $\boldsymbol{x}_{L} \in L$ be given and $d_{K, \sigma}$ (resp. $d_{L, \sigma}$ ) the distance from $x_{K}$ resp. $x_{L}$ ) to $\sigma$ (see Figure 1(a)). Let $\boldsymbol{y}_{K}$ and $\boldsymbol{y}_{L} \in \sigma$ such that $\boldsymbol{x}_{K}=-d_{K, \sigma} \boldsymbol{n}_{K L}+\boldsymbol{y}_{K}$ and $\boldsymbol{x}_{L}=d_{L, \sigma} \boldsymbol{n}_{K L}+\boldsymbol{y}_{L}$. Let $\boldsymbol{y}_{\sigma} \in \sigma$ (called the harmonic averaging point) be defined by

$$
\boldsymbol{y}_{\sigma}=\frac{\lambda_{L} d_{K, \sigma} \boldsymbol{y}_{L}+\lambda_{K} d_{L, \sigma} \boldsymbol{y}_{K}}{\lambda_{L} d_{K, \sigma}+\lambda_{K} d_{L, \sigma}}+\frac{d_{K, \sigma} d_{L, \sigma}}{\lambda_{L} d_{K, \sigma}+\lambda_{K} d_{L, \sigma}}\left(\boldsymbol{\lambda}_{K}^{\sigma}-\boldsymbol{\lambda}_{L}^{\sigma}\right),
$$

denoting by $\lambda_{K}=\boldsymbol{n}_{K L} \cdot \Lambda_{K} \boldsymbol{n}_{K L}, \lambda_{K}^{\sigma}=\left(\Lambda_{K}-\lambda_{K} \operatorname{Id}\right) \boldsymbol{n}_{K L}, \lambda_{L}=\boldsymbol{n}_{K L} \cdot \Lambda_{L} \boldsymbol{n}_{K L}$ and $\boldsymbol{\lambda}_{L}^{\sigma}=\left(\Lambda_{L}-\lambda_{L} \mathrm{Id}\right) \boldsymbol{n}_{K L}$. Then the following averaging formula holds, for all functions $u$ defined on $\mathbb{R}^{d}$, affine in $K$ and $L$, such that $u$ is continuous on $\sigma$, and such that $\Lambda_{K} \nabla u_{\mid K} \cdot \boldsymbol{n}_{K L}=\Lambda_{L} \nabla u_{\mid L} \cdot \boldsymbol{n}_{K L}$ :

$$
u\left(\boldsymbol{y}_{\sigma}\right)=\frac{\lambda_{L} d_{K, \sigma} u\left(\boldsymbol{x}_{L}\right)+\lambda_{K} d_{L, \sigma} u\left(\boldsymbol{x}_{K}\right)}{\lambda_{L} d_{K, \sigma}+\lambda_{K} d_{L, \sigma}} .
$$

Let us sketch the proof of the lemma. We denote by $\boldsymbol{G}_{K}$ the gradient of $u$ in $K$, with $\boldsymbol{G}_{K}=g_{K} \boldsymbol{n}_{K L}+\boldsymbol{G}_{K}^{\sigma}, \boldsymbol{G}_{K}^{\sigma} \cdot \boldsymbol{n}_{K L}=0$ and by $\boldsymbol{G}_{L}=g_{L} \boldsymbol{n}_{K L}+\boldsymbol{G}_{L}^{\sigma}, \boldsymbol{G}_{L}^{\sigma} \cdot \boldsymbol{n}_{K L}=0$ the gradient of $u$ in $L$. The continuity property of $u$ on $\sigma$ first leads to $\boldsymbol{G}_{K}^{\sigma}=$ $\boldsymbol{G}_{L}^{\sigma}=\boldsymbol{g}^{\sigma}$ and then to $d_{K, \sigma} g_{K}+d_{L, \sigma} g_{L}=u\left(\boldsymbol{x}_{L}\right)-u\left(\boldsymbol{x}_{K}\right)+\left(\boldsymbol{y}_{K}-\boldsymbol{y}_{L}\right) \cdot \boldsymbol{g}^{\sigma}$, and the condition $\Lambda_{K} \boldsymbol{G}_{K} \cdot \boldsymbol{n}_{K L}=\Lambda_{L} \boldsymbol{G}_{L} \cdot \boldsymbol{n}_{K L}$ can be written $g_{K} \lambda_{K}-g_{L} \lambda_{L}=\boldsymbol{g}^{\sigma} .\left(\boldsymbol{\lambda}_{L}^{\sigma}-\boldsymbol{\lambda}_{K}^{\sigma}\right)$. 
Expressing $g_{K}$ with respect to $\boldsymbol{g}^{\sigma}$ and using $u(\boldsymbol{y})=u\left(\boldsymbol{x}_{K}\right)+\boldsymbol{G}_{K} \cdot\left(\boldsymbol{y}-\boldsymbol{x}_{K}\right)$, for all $\boldsymbol{y} \in \sigma$, allows us to write that

$$
\begin{aligned}
u(\boldsymbol{y})= & u\left(\boldsymbol{x}_{K}\right) \\
& +d_{K, \sigma} \frac{\lambda_{L}\left(u\left(\boldsymbol{x}_{L}\right)-u\left(\boldsymbol{x}_{K}\right)+\left(\boldsymbol{y}_{K}-\boldsymbol{y}_{L}\right) \cdot \boldsymbol{g}^{\sigma}\right)+d_{L, \sigma} \boldsymbol{g}^{\sigma} \cdot\left(\boldsymbol{\lambda}_{L}^{\sigma}-\boldsymbol{\lambda}_{K}^{\sigma}\right)}{\lambda_{L} d_{K, \sigma}+\lambda_{K} d_{L, \sigma}} \\
& +\left(\boldsymbol{y}-\boldsymbol{y}_{K}\right) \cdot \boldsymbol{g}^{\sigma} .
\end{aligned}
$$

The point $\boldsymbol{y}_{\sigma}$ is then defined as the unique point $\boldsymbol{y} \in \sigma$ such that the preceding expression no longer depends on $\boldsymbol{g}^{\sigma}$, and the resulting expression for $u\left(\boldsymbol{y}_{\sigma}\right)$ follows.

\section{Definition of the SCheme}

We consider general polygonal, possibly non conforming, meshes of $\Omega$ (as in [4]). Let $\mathcal{T}$ be the set of control volumes, that are disjoint open polygonal subsets of $\Omega$ such that $\bigcup_{K \in \mathcal{T}} \bar{K}=\bar{\Omega}$. We denote by $\Lambda_{K}$ the mean value of $\Lambda$ in $K \in \mathcal{T}$. Let $\mathcal{E}$ be the set of edges of the mesh; we denote by $\mathcal{E}_{K}$ the set of the edges of any $K \in \mathcal{T}$. Let $\mathcal{P}=\left\{\boldsymbol{x}_{K}, K \in \mathcal{T}\right\}$ be the set of the so-called "centres" of the control volumes, which are the approximation points. We assume that, for all $K \in \mathcal{T}, K$ is star-shaped with respect to $\boldsymbol{x}_{K}$. Let $\mathcal{V}$ be the set of the vertices of the mesh, and let $\mathcal{V}_{\sigma}\left(\right.$ resp. $\left.\mathcal{V}_{K}\right)$ be the set of the vertices of any $\sigma \in \mathcal{E}$ (resp. $K \in \mathcal{T}$ ). For any edge $\sigma \subset \partial \Omega$, we denote by $\boldsymbol{y}_{\sigma}$ its centre point. For any interior edge $\sigma$, we denote by $\boldsymbol{y}_{\sigma}$ the harmonic averaging point defined by (2) if this point is interior to $\sigma$ (we then denote by $\mathcal{E}_{\sharp}$ the set of such edges), and by the centre of $\sigma$ otherwise.

Remark 1 (Harmonic averaging points and meshes). The condition $\sigma \in \mathcal{E}_{\sharp}$ generally holds for meshes which are not "too distorted". It holds in particular if $\left[\boldsymbol{x}_{K}, \boldsymbol{x}_{L}\right] \cap \sigma$ is an interior point to $\sigma$ in the case of identical diffusion matrices $\Lambda_{K}$ and $\Lambda_{L}$. It also holds if the orthogonal projections of $\boldsymbol{x}_{K}$ and $\boldsymbol{x}_{L}$ on $\sigma$ are interior points to $\sigma$ in the case of isotropic diffusion matrices $\Lambda_{K}$ and $\Lambda_{L}$. We show in Figure 2 below an example of a distorted mesh for which there exists one edge that does not satisfy $\sigma \in \mathcal{E}_{\sharp}$ in the case of a homogeneous medium.

Let $X_{\mathcal{T}}$ be the set of all families $u=\left\{u_{K}, u_{\sigma}, u_{\sigma, s}, K \in \mathcal{T}, \sigma \in \mathcal{E}, s \in \mathcal{V}_{\sigma}\right.$, with $u_{\sigma}=u_{\sigma, s}=0$ for $\left.\sigma \subset \partial \Omega\right\}$. Let $X_{\mathcal{T}}^{\sharp}$ be the subset of all $u \in X_{\mathcal{T}}$ such that, for any $\sigma \in \mathcal{E}_{\sharp}, u_{\sigma}$ is defined by:

$$
u_{\sigma}=\frac{\lambda_{L} d_{K, \sigma} u_{L}+\lambda_{K} d_{L, \sigma} u_{K}}{\lambda_{L} d_{K, \sigma}+\lambda_{K} d_{L, \sigma}}
$$

For $u \in X_{\mathcal{T}}$, we define by $\Pi_{\mathcal{T}} u$ the piecewise constant function defined on $\Omega$, with the constant value $u_{K}$ in $K \in \mathcal{T}$. For all $K \in \mathcal{T}$ and $s \in \mathcal{V}_{K}$, we denote by $K_{\boldsymbol{s}}$ the open quadrilateral domain, the boundary of which is composed of the line segments belonging to the set: $\mathcal{E}_{K, \boldsymbol{s}}=\left\{\left[\boldsymbol{x}_{K}, \boldsymbol{y}_{\sigma}\right],\left[\boldsymbol{x}_{K}, \boldsymbol{y}_{\sigma^{\prime}}\right],\left[\boldsymbol{s}, \boldsymbol{y}_{\sigma}\right],\left[\boldsymbol{s}, \boldsymbol{y}_{\sigma^{\prime}}\right]\right\}$, where $\sigma, \sigma^{\prime} \in \mathcal{E}_{K}$ are the two edges of $K$ with vertex $\boldsymbol{s}$ (see figure 1(b)). For all $\epsilon \in \mathcal{E}_{K, \boldsymbol{s}}$, we denote by $\boldsymbol{n}_{K, \boldsymbol{s}}^{\epsilon}$ the normal vector to $\epsilon$ outward to $K_{\boldsymbol{s}}$. For any $u \in X_{\mathcal{T}}$, we denote by $u_{K, s}^{\epsilon}$, for any $\epsilon \in \mathcal{E}_{K, s}$, the values defined by:

$u_{K, \boldsymbol{s}}^{\epsilon}=\frac{u_{K}+u_{\tau}}{2}$ if $\epsilon=\left[\boldsymbol{x}_{K}, \boldsymbol{y}_{\tau}\right]$ and $u_{K, \boldsymbol{s}}^{\epsilon}=u_{\tau, \boldsymbol{s}}$ if $\epsilon=\left[\boldsymbol{s}, \boldsymbol{y}_{\tau}\right]$ for $\tau=\sigma$ or $\sigma^{\prime}$. 
We then define a piecewise discrete gradient $\nabla_{\mathcal{T}} u$ by its constant values $\nabla_{K, \boldsymbol{s}} u \in \mathbb{R}^{2}$ on the subcells $K_{s}$ :

$$
\left|K_{\boldsymbol{s}}\right| \nabla_{K, \boldsymbol{s}} u=\sum_{\epsilon \in \mathcal{E}_{K, \boldsymbol{s}}}|\epsilon|\left(u_{K, \boldsymbol{s}}^{\epsilon}-u_{K}\right) \boldsymbol{n}_{K, \boldsymbol{s}}^{\epsilon} .
$$

This discrete gradient satisfies the following two fundamental properties, which are also those of the cell piecewise constant gradient used in [6]:

(1) consistency of $\nabla_{\mathcal{T}} \varphi_{\mathcal{T}}$ with $\nabla \varphi$ for a class of regular functions $\varphi$ and their interpolation $\varphi_{\mathcal{T}}$ in $X_{\mathcal{T}}$,

(2) weak convergence, as the size of the mesh tends to 0 , of $\nabla_{\mathcal{T}} u$ to $\nabla \bar{u}$ such that $u \rightarrow \bar{u}$ under suitable estimates on $u \in X_{\mathcal{T}}$.

Both properties follow from the choice (6) and the fact that, for any $\mathbf{w} \in \mathbb{R}^{2}$, one has: $\left|K_{\boldsymbol{s}}\right| \mathbf{w}=\int_{\partial K_{s}} \mathbf{w} \cdot \boldsymbol{x} \boldsymbol{n}_{K, \boldsymbol{s}}$; property (i) is also a consequence of the choice (4). For all $u, v \in X_{\mathcal{T}}$, we define a discrete inner product, expected to approximate the bilinear form $(u, v) \mapsto \int_{\Omega} \Lambda \nabla u \cdot \nabla v$. A natural choice would be $[u, v]=\sum_{K \in \mathcal{T}} \sum_{s \in \mathcal{V}_{K}}[u, v]_{K, \boldsymbol{s}}$, where $[u, v]_{K, \boldsymbol{s}}=\left|K_{\boldsymbol{s}}\right| \Lambda_{K} \nabla_{K, \boldsymbol{s}} u \cdot \nabla_{K, \boldsymbol{s}} v$. However, this choice yields a non-coercive bilinear form, and therefore, as in [6], we stabilise it by choosing rather:

$$
\begin{gathered}
\langle u, v\rangle=\sum_{K \in \mathcal{T}} \sum_{s \in \mathcal{V}_{K}}\langle u, v\rangle_{K, \boldsymbol{s}}, \\
\text { with }\langle u, v\rangle_{K, \boldsymbol{s}}=\left|K_{\boldsymbol{s}}\right| \Lambda_{K} \nabla_{K, \boldsymbol{s}} u \cdot \nabla_{K, \boldsymbol{s}} v+\sum_{\tau=\sigma, \sigma^{\prime}} \alpha_{K \tau} R_{K, \boldsymbol{s}}^{\tau} u R_{K, \boldsymbol{s}}^{\tau} v,
\end{gathered}
$$

where $\alpha_{K \tau}>0, R_{K, \boldsymbol{s}}^{\tau} u=u_{\tau}-u_{K}-\nabla_{K, \boldsymbol{s}} u \cdot\left(\boldsymbol{y}_{\tau}-\boldsymbol{x}_{K}\right)$, for $\tau=\sigma$ and $\sigma^{\prime}$. The scheme is then defined by

$$
\text { find } u \in X_{\mathcal{T}}^{\sharp} \text {, such that for any } v \in X_{\mathcal{T}}^{\sharp},\langle u, v\rangle=\int_{\Omega} f(\boldsymbol{x}) \Pi_{\mathcal{T}} v(\boldsymbol{x}) \mathrm{d} \boldsymbol{x} .
$$

Note that $\langle u, v\rangle_{K, s}$ can be written under the form

$$
\langle u, v\rangle_{K, \boldsymbol{s}}=\sum_{\epsilon \in \mathcal{E}_{K, \boldsymbol{s}}} \sum_{\epsilon^{\prime} \in \mathcal{E}_{K, \boldsymbol{s}}} A_{K, \boldsymbol{s}}^{\epsilon^{\prime} \epsilon}\left(u_{K, \boldsymbol{s}}^{\epsilon^{\prime}}-u_{K}\right)\left(v_{K, \boldsymbol{s}}^{\epsilon}-v_{K}\right),
$$

where the $4 \times 4$ matrix $A_{K, s}$ is symmetric. We may then define

$$
F_{K, \boldsymbol{s}}^{\epsilon}(u)=\sum_{\epsilon^{\prime} \in \mathcal{E}_{K, \boldsymbol{s}}} A_{K, \boldsymbol{s}}^{\epsilon^{\prime} \epsilon}\left(u_{K, \boldsymbol{s}}^{\epsilon^{\prime}}-u_{K}\right),
$$

and write that

$$
\langle u, v\rangle_{K, \boldsymbol{s}}=\sum_{\epsilon \in \mathcal{E}_{K, \boldsymbol{s}}} F_{K, \boldsymbol{s}}^{\epsilon}(u)\left(v_{K, \boldsymbol{s}}^{\epsilon}-v_{K}\right) .
$$

We get from (8) for an edge $\sigma=K \mid L$ common to $K$ and $L$ and for $s \in \mathcal{V}_{\sigma}$, setting $v_{\sigma, s}=1$ and all the other values of $v$ to zero,

$$
F_{K, \boldsymbol{s}}^{\epsilon}(u)+F_{L, s}^{\epsilon}(u)=0 \text { if } \epsilon=\left[\boldsymbol{s}, \boldsymbol{y}_{\sigma}\right] .
$$

For a given $s \in \mathcal{V}$, denoting by $\mathcal{E}_{s}$ the set of all $\sigma \in \mathcal{E}$ such that $s \in \mathcal{V}_{\sigma}$, one may show that the subsystem with unknowns $\left(u_{\sigma, s}\right)_{\sigma \in \mathcal{E}_{s}}$ is invertible, so that these latter unknowns can be eliminated from the system of equations (9) written for all $\sigma \in \mathcal{E}_{\boldsymbol{s}}$ : the values $u_{\sigma, s}$ may thus be written as linear combinations of the values $u_{K}, u_{\sigma}$ for $s \in \mathcal{V}_{K}$ and $s \in \mathcal{V}_{\sigma}$. Hence, in the case where all interior edges satisfy $\sigma \in \mathcal{E}_{\sharp}$, this elimination provides a cell-centred scheme which has the nine-point stencil on 
structured quadrilateral meshes (note that this elimination is also a feature of the O-scheme [2]).

Reordering the terms in (7) and using (5), we may write

$$
\langle u, v\rangle=\sum_{\sigma \in \mathcal{E}}\left(T_{\sigma}^{(1)}(u, v)+T_{\sigma}^{(2)}(u, v)\right),
$$

where for an edge $\sigma$ common to control volumes $K$ and $L$,

$$
T_{\sigma}^{(1)}(u, v)=\sum_{\boldsymbol{s} \in \mathcal{V}_{\sigma}}\left(F_{K, \boldsymbol{s}}^{\left[\boldsymbol{x}_{K}, \boldsymbol{y}_{\sigma}\right]}(u) \frac{1}{2}\left(v_{\sigma}-v_{K}\right)+F_{L, \boldsymbol{s}}^{\left[\boldsymbol{x}_{L}, \boldsymbol{y}_{\sigma}\right]}(u) \frac{1}{2}\left(v_{\sigma}-v_{L}\right)\right),
$$

and

$$
T_{\sigma}^{(2)}(u, v)=\sum_{\boldsymbol{s} \in \mathcal{V}_{\sigma}}\left(F_{K, \boldsymbol{s}}^{\left[\boldsymbol{s}, \boldsymbol{y}_{\sigma}\right]}(u)\left(v_{\sigma, \boldsymbol{s}}-v_{K}\right)+F_{L, \boldsymbol{s}}^{\left[\boldsymbol{s}, \boldsymbol{y}_{\sigma}\right]}(u)\left(v_{\sigma, \boldsymbol{s}}-v_{L}\right)\right),
$$

and for an edge $\sigma$ of $K$ located on the boundary $\partial \Omega$,

$$
\begin{gathered}
T_{\sigma}^{(1)}(u, v)=\sum_{\boldsymbol{s} \in \mathcal{V}_{\sigma}} F_{K, \boldsymbol{s}}^{\left[\boldsymbol{x}_{K}, \boldsymbol{y}_{\sigma}\right]}(u) \frac{1}{2}\left(v_{\sigma}-v_{K}\right)=F_{K, \sigma}^{(1)}(u) v_{K} \\
\text { with } F_{K, \sigma}^{(1)}(u)=-\frac{1}{2} \sum_{\boldsymbol{s} \in \mathcal{V}_{\sigma}} F_{K, \boldsymbol{s}}^{\left[\boldsymbol{x}_{K}, \boldsymbol{y}_{\sigma}\right]}(u),
\end{gathered}
$$

and

$$
\begin{gathered}
T_{\sigma}^{(2)}(u, v)=\sum_{\boldsymbol{s} \in \mathcal{V}_{\sigma}} F_{K, \boldsymbol{s}}^{\left[\boldsymbol{s}, \boldsymbol{y}_{\sigma}\right]}(u)\left(v_{\sigma, \boldsymbol{s}}-v_{K}\right)=F_{K, \sigma}^{(2)}(u) v_{K} \\
\text { with } F_{K, \sigma}^{(2)}(u)=-\sum_{\boldsymbol{s} \in \mathcal{V}_{\sigma}} F_{K, \boldsymbol{s}}^{\left[\boldsymbol{s}, \boldsymbol{y}_{\sigma}\right]}(u) .
\end{gathered}
$$

Now, for an edge $\sigma$ common to control volumes $K$ and $L$, thanks to (9),

$$
\begin{gathered}
T_{\sigma}^{(2)}(u, v)=F_{K, L}^{(2)}(u)\left(v_{K}-v_{L}\right) \\
\text { with } F_{K, L}^{(2)}(u)=-\sum_{\boldsymbol{s} \in \mathcal{V}_{\sigma}} F_{K, \boldsymbol{s}}^{\left[\boldsymbol{s}, \boldsymbol{y}_{\sigma}\right]}(u)=\sum_{\boldsymbol{s} \in \mathcal{V}_{\sigma}} F_{L, \boldsymbol{s}}^{\left[\boldsymbol{s}, \boldsymbol{y}_{\sigma}\right]}(u) .
\end{gathered}
$$

Let us then turn to the term $T_{\sigma}^{(1)}(u, v)$. In the case where $\sigma \in \mathcal{E}_{\sharp}$, we have from (4): $v_{\sigma}-v_{K}=\frac{\lambda_{L} d_{K, \sigma}}{\lambda_{L} d_{K, \sigma}+\lambda_{K} d_{L, \sigma}}\left(v_{L}-v_{K}\right)$ and $v_{\sigma}-v_{L}=\frac{\lambda_{K} d_{L, \sigma}}{\lambda_{L} d_{K, \sigma}+\lambda_{K} d_{L, \sigma}}\left(v_{K}-v_{L}\right)$,

which leads to

$$
\begin{gathered}
T_{\sigma}^{(1)}(u, v)=F_{K, L}^{(1)}(u)\left(v_{K}-v_{L}\right) \\
\text { with } F_{K, L}^{(1)}(u)=\sum_{\boldsymbol{s} \in \mathcal{V}_{\sigma}} \frac{-\lambda_{L} d_{K, \sigma} F_{K, \boldsymbol{s}}^{\left[\boldsymbol{s}, \boldsymbol{y}_{\sigma}\right]}(u)+\lambda_{K} d_{L, \sigma} F_{L, \boldsymbol{s}}^{\left[\boldsymbol{s}, \boldsymbol{y}_{\sigma}\right]}(u)}{2\left(\lambda_{L} d_{K, \sigma}+\lambda_{K} d_{L, \sigma}\right)} .
\end{gathered}
$$

In the case where $\sigma \notin \mathcal{E}_{\sharp}$, taking in (8) $v \in X_{\mathcal{T}}^{\sharp}$ such that $v_{\sigma}=1$ and all other components set to 0 , we get that $\frac{1}{2} \sum_{\boldsymbol{s} \in \mathcal{V}_{\sigma}}\left(F_{K, \boldsymbol{s}}^{\left[\boldsymbol{x}_{K}, \boldsymbol{y}_{\sigma}\right]}(u)+F_{L, \boldsymbol{s}}^{\left[\boldsymbol{x}_{L}, \boldsymbol{y}_{\sigma}\right]}(u)\right)=0$, which leads to

$$
\begin{gathered}
T_{\sigma}^{(1)}(u, v)=F_{K, L}^{(1)}(u)\left(v_{K}-v_{L}\right) \\
\text { with } F_{K, L}^{(1)}(u)=-\frac{1}{2} \sum_{\boldsymbol{s} \in \mathcal{V}_{\sigma}} F_{K, \boldsymbol{s}}^{\left[\boldsymbol{x}_{K}, \boldsymbol{y}_{\sigma}\right]}(u)=\frac{1}{2} \sum_{\boldsymbol{s} \in \mathcal{V}_{\sigma}} F_{L, \boldsymbol{s}}^{\left[\boldsymbol{x}_{L}, \boldsymbol{y}_{\sigma}\right]}(u) .
\end{gathered}
$$




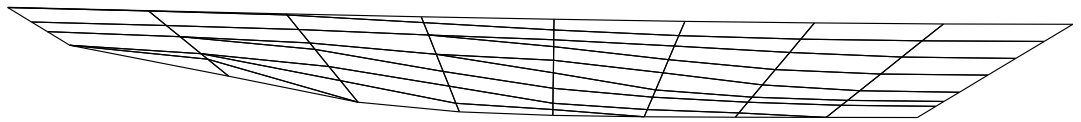

Figure 2. Example of mesh

Hence the scheme (8) is a finite volume scheme: indeed, taking $v$ such that $v_{K}=1$ and all other components set to 0 in (8), one has:

$$
\forall K \in \mathcal{T}, \sum_{\substack{\sigma \in \mathcal{E}_{K} \\ \sigma=K \mid L}} F_{K, L}(u)+\sum_{\substack{\sigma \in \mathcal{E}_{K} \\ \sigma \subset \partial \Omega}} F_{K, \sigma}(u)=\int_{K} f(\boldsymbol{x}) \mathrm{d} x
$$

where $F_{K, \sigma}(u)=F_{K, \sigma}^{(1)}(u)+F_{K, \sigma}^{(2)}(u)$ and $F_{K, L}(u)=F_{K, L}^{(1)}(u)+F_{K, L}^{(2)}(u)=-F_{L, K}(u)$.

Finally, let us mention that he proof of convergence of the scheme (8) may be adapted from that of $[6]$.

\section{Numerical Results}

We tested the scheme for some of the cases described in the benchmark [5], in particular those with anisotropy and heterogeneity such as tests cases 5 and 6 (geological barrier and drain), and, as expected, the results are exact since the solution is piecewise affine in these cases. We have also run test case 5 (heterogeneous rotating anisotropy). An order 2 of convergence is then observed on the $L^{2}$-norm of the unknown. The finest mesh that we used for this test has $640 \times 640$ grid blocks, computed within a few minutes on a PC. A direct solver could be used, with numbering the unknowns using classical methods holding for 9-point stencils [3].

We also consider a test case with a mesh inspired form those used in geological studies (see figure 4 ). We take $\Lambda=\operatorname{diag}(0.1,1)$ and $f$ such that the exact solution be given by $u(x, y)=\sin (\pi x) \sin (\pi y)$. 2:

We get the following results when refining the mesh (mesh 1) depicted in Figure

\begin{tabular}{|c|c|c|c|c|c|}
\hline & mesh 1 & mesh 2 & mesh 3 & mesh 4 & mesh 5 \\
\hline$\# \mathcal{T}$ & 62 & 302 & 1357 & 5363 & 21031 \\
\hline$\#$ hybrid edges & 1 & 3 & 6 & 10 & 17 \\
\hline$L^{2}$-error & $9.1510^{-3}$ & $3.0710^{-3}$ & $9.3010^{-4}$ & $2.6610^{-4}$ & $6.8910^{-5}$ \\
\hline
\end{tabular}

These results confirm the expected numerical convergence.

\section{REFERENCES}

[1] I. Aavatsmark, T. Barkve, O. Boe, and T. Mannseth. Discretization on non-orthogonal, quadrilateral grids for inhomogeneous, anisotropic media. J. Comput. Phys., 127(1):2-14, 1996.

[2] I. Aavatsmark, T. Barkve, O. Boe, and T. Mannseth. Discretization on unstructured grids for inhomogeneous, anisotropic media. part i: Derivation of the methods. SIAM Journal on Sc. Comp., 19:1700-1716, 1998.

[3] I.S. Duff, A.M. Erisman, and J.K. Reid. Direct methods for sparse matrices. Monographs on Numerical Analysis. Oxford: Clarendon Press. XIII, 341 p. Ł 25.00 , 1986.

[4] R. Eymard, T. Gallouët, and R. Herbin. A new finite volume scheme for anisotropic diffusion problems on general grids: convergence analysis. C. R., Math., Acad. Sci. Paris, 344(6):403406, 2007. 
[5] R. Eymard, T. Gallouët, and R. Herbin. Benchmark on anisotropic problems, SUSHI: a scheme using stabilization and hybrid interfaces for anisotropic heterogeneous diffusion problems. In R. Eymard and J.-M. Hérard, editors, Finite Volumes for Complex Applications V, pages 801-814. Wiley, 2008.

[6] R. Eymard, T. Gallouët, and R. Herbin. Discretisation of heterogeneous and anisotropic diffusion problems on general non-conforming meshes, sushi: a scheme using stabilisation and hybrid interfaces. to appear in IMAJNA, see also http://hal.archives-ouvertes.fr/docs/ 00/21/18/28/PDF/suchi.pdf,, 2008.

[7] R. Herbin and F. Hubert. Benchmark on discretization schemes for anisotropic diffusion problems on general grids for anisotropic heterogeneous diffusion problems. In R. Eymard and J.-M. Hérard, editors, Finite Volumes for Complex Applications V, pages 659-692. Wiley, 2008.

Institut FranÇAis du PÉTrole

E-mail address: leo.agelas@ifp.fr

UnIVERsité PARIS-Est

E-mail address: robert.eymard@univ-mlv.fr

Université DE Provence

E-mail address: herbin@cmi.univ-mrs.fr 